\title{
Oxymatrine protects against myocardial injury via inhibition of JAK2/STAT3 signaling in rat septic shock
}

\author{
MINGHAO ZHANG $^{1 *}$, XIUYU WANG ${ }^{1 *}$, XIUMEI WANG ${ }^{2 *}$, XIAOLIN HOU ${ }^{3 *}$, \\ PENG TENG ${ }^{1}$, YIDENG JIANG ${ }^{1}$, LINNA ZHANG $^{1}$, XIAOLING YANG $^{1}$, JUE TIAN $^{1}$, \\ GUIZHONG LI ${ }^{1}$, JUN CAO ${ }^{1}$, HUA XU ${ }^{1}$, YUNHONG LI ${ }^{1}$ and YIN WANG ${ }^{1}$

\begin{abstract}
${ }^{1}$ Department of Pathophysiology and Neurobiology, Basic Medical College, Center of Scientific Technology, Ningxia Medical University, Yinchuan 750004; ${ }^{2}$ Department of Pathology, Taiyuan Maternal and Child Health Hospital, Taiyuan 030012;

${ }^{3}$ Department of Neurology, General Hospital of Ningxia Medical University, Yinchuan 750004, P.R. China
\end{abstract}

Received November 22, 2012; Accepted January 21, 2013

DOI: $10.3892 / \mathrm{mmr} .2013 .1315$

\begin{abstract}
Oxymatrine (OMT), an alkaloid extracted from Sophora japonica (kushen), is used to treat inflammatory diseases and various types of cancer in traditional Chinese medicine. However, the cellular and molecular mechanisms underlying the anti-inflammatory activity of OMT remain poorly understood. The present study explored the protective effect of OMT on myocardial injury in rats with septic shock by inhibiting the activation of the janus kinase-signal transducer and activator of transcription (JAK/STAT) signaling pathway. OMT treatment was found to significantly inhibit the activation of JAK2 and STAT3 in myocardial tissue. It also attenuated the expression of pro-inflammatory cytokines, including interleukin- $1 \beta$ and tumor necrosis factor- $\alpha$. In addition, OMT exhibited anti-inflammatory properties as heart function and myocardial contractility was improved and pathological and ultrastructural injury was prevented in myocardial tissue induced by septic shock. The results indicate that OMT exhibits substantial therapeutic potential for the treatment of septic shock-induced myocardial injury through inhibition of the JAK2/STAT3 signaling pathway.
\end{abstract}

\section{Introduction}

Septic shock is a result of severe sepsis, which is produced by microorganisms and their toxins. These cause low blood pressure, hypoxemia, metabolic acidosis, systemic inflammatory reaction and multiple organ dysfunctions characteristic

Correspondence to: Professor Yin Wang, Department of Neurobiology, Basic Medical College, Ningxia Medical University, 1160 Shengli Street, Yinchuan 750004, P.R. China

E-mail: yin-wang@hotmail.com

*Contributed equally

Key words: oxymatrine, septic shock, myocardial injury, JAK2/STAT3 of complex pathophysiological processes. Septic shock affects critical care patients and is one of the main causes of mortality (1). Myocardial dysfunction is a common complication during sepsis and significantly contributes to the mortality of patients with septic shock (2). Previous studies on septic shock have concentrated on the generation of inflammatory mediators, including tumor necrosis factor- $\alpha$ (TNF- $\alpha$ ) and interleukin-1 $\beta$ (IL-1 $\beta$ ) and damage to liver, lung and kidney tissues (3). Myocardial depression is a well-recognized manifestation of organ dysfunction in sepsis. However, the various mechanisms underlying septic cardiac dysfunction remain unclear and none of the current therapeutic strategies designed specifically to target myocardial dysfunction have proven to be effective in patients with septic shock (4).

The janus kinase-signal transduction and activator of transcription (JAK-STAT) signaling pathway is one of the best understood signal transduction cascades and is essential for cytokine receptor signaling involved in immune and inflammatory responses. JAK-STAT signaling molecules bind specific nucleotide sequences in the gene promoter region of cytokines and inflammatory cytokines, including TNF- $\alpha$, IL-1 $\beta$ and IL-6, inducing gene expression $(5,6)$. Our previous study revealed that the JAK/STAT signaling pathway was activated and the expression of TNF- $\alpha$ and IL- 6 increased in rat lung tissue with sepsis (7). Inhibition of the JAK/STAT pathway attenuated multiple organ dysfunction in rats with sepsis (8). These observations indicate that the activation of the JAK/STAT signaling pathway is associated with the occurrence and development of systemic inflammatory reaction. Thus, preventing the activation of JAK/STAT signaling pathways to reduce inflammation may have clinical benefits for patients suffering from septic shock.

OMT, the major active component of the traditional Chinese medicine, kushen, has been well studied and its anti-inflammatory activity has been demonstrated in experimental animal models and clinical studies $(9,10)$. OMT exhibits a variety of biological activities, including clearance of heat and toxic material, anti-inflammatory, inhibition of liver fibrosis, elevation of white blood cells, coxsackie virus resistance and regulation of immunity and other aspects of pharmacological action $(11,12)$. It has been reported that OMT may reduce the generation of oleic 
acid in rats induced by acute lung injury by inhibiting the $\mathrm{p} 38$ MAPK signaling pathway and the expression of TNF- $\alpha$ (13). Lung tissue damage in mice with endotoxemia has been identified to be inhibited by OMT and this mechanism is associated with regulation of the LPS recognition receptor expression and downregulation of the expression of downstream inflammatory factors (14). These observations indicate that OMT plays a vital role in anti-inflammatory reactions and are consistent with our hypothesis that OMT may prevent myocardial damage with infectious shock by inhibiting the activation of JAK/STAT signaling pathways.

In the present study, cecal ligation and puncture (CLP) was performed in a rat model to examine the effect of OMT on inhibition of the JAK/STAT signaling pathway in cardiac muscle injury with septic shock.

\section{Materials and methods}

Chemicals. Oxymatrine was purchased from Ningxia Qi Yuan Pharmaceutical Co. (Yinchuan, China). Dexamethasone was from Hubei Day Drug Pharmaceutical Co. (Hubei, China). Coomassie Brilliant Blue protein quantification kit was purchased from Nanjing Jiancheng Bioengineering Company (Nanjing, China). The ${ }^{125} \mathrm{I}$ tumor necrosis factor- $\alpha$ and ${ }^{125}$ I interleukin- $1 \beta$ radiation immunoassay kits were purchased from the Beijing Chemclin Bioengineering Company (Beijing, China). TRIzol was obtained from Invitrogen Life Technologies (Carlsbad, CA. USA). PrimeScript RT reagent kit was from Takara Bio (Takara Bio, Inc., Dalian, China). RT-PCR kit was from Promega (Promega Corporation, Madison, WI, USA). Rat monoclonal antibodies against JAK2 and STAT3 were from Santa Cruz Biotechnology, Inc. (Santa Cruz, CA, USA) and IP cell lystates and the BCA protein concentration assay kit were from Jiangsu Green Biotechnology Company (Jiangsu, China).

Animals. Animal studies were approved by the Ningxia Medical University Animal Care Committee (Ningxia, China). Male Sprague-Dawley rats, specific pathogen free, weighing 200-250 g, were obtained from the Animal Center of Ningxia Medical University [SCXK (Ning) 2005-001]. Rats were randomly divided into 7 groups $(n=8)$ : sham surgery, OMT control, CLP model, positive control [CLP + dexamethasone (DEX), $10 \mathrm{mg} / \mathrm{kg}$ ] and CLP + OMT 52, 26 and $13 \mathrm{mg} / \mathrm{kg}$. The septic shock model was induced by CLP as described previously (15). Rats received tail vein injection with drugs (volume, $5 \mathrm{ml} / \mathrm{kg}$ ). In the sham-operated and OMT control groups, the rats received intravenous injection with normal saline and OMT $(26 \mathrm{ml} / \mathrm{kg}$ ) and the CLP group received normal saline $(26 \mathrm{ml} / \mathrm{kg}$ ) only. Following treatment, the cecum was exposed under sterile conditions. Postoperative monitoring rat tail artery pressure was performed, and rat blood pressure dropped to $2 / 3$ of base blood pressure and pulse pressure $<20 \mathrm{mmHg}$ was the standard used to judge the success of the sepsis model.

Cardiac function and histological analyses. To examine cardiac function, the right common carotid artery was used to determine the rat heart rate (HR), mean arterial pressure (MAP), left intraventricular pressure change rate (LVdp/dt max), left ventricular end systolic pressure (LVESP) and left ventricular end diastolic pressure (LVEDP) by inserting the cardiac catheterization. The heart was then removed for histological analysis. Apical tissue blocks $\left(\sim 2 \mathrm{~mm}^{3}\right)$ were collected and fixed in $10 \%$ formalin, then embedded in paraffin. Following haematoxylin-eosin staining, pathological changes of myocardial tissue were observed under the light microscope. Additional myocardial tissues $\sim 2 \mathrm{~mm}^{3}$ were placed in $2 \%$ glutaraldehyde and sectioned as electron microscopy specimens to determine ultrastructural changes.

RT-PCR analysis. Total RNA was prepared using TRIzol and was reverse-transcribed to cDNA using a PrimeScript RT reagent kit. RT-PCR was performed using a commercially available kit as follows: 35 cycles of denaturation at $94^{\circ} \mathrm{C}$ for $60 \mathrm{sec}$, annealing at $58^{\circ} \mathrm{C}$ for $60 \mathrm{sec}$ and extension at $72^{\circ} \mathrm{C}$ for $50 \mathrm{sec} . \beta$-actin was used as an internal control to evaluate relative expression of TNF- $\alpha$ and IL-1 $\beta$. The primers used were: TNF- $\alpha$ (355 bp) 5'-CAATGGCATGGATCTCAAAG-3' and 5'-CAGAGCAATGACTCCAAAGT-3', IL-1 $\beta$ (399 bp) 5'-AGAAGCTGTGGCAGCTACCT-3' and 5'-TTGGGA TCCACACTCTCCAG-3', $\beta$-actin (299 bp) 5'-AGGTGAGAG GGAAATCGTGCG-3' and 5'-GTGCCACCAGACAGC ACTGTGC-3'.

Western blot analysis. Total protein concentration was measured using a BCA kit. Equal amounts of protein $(40 \mu \mathrm{g})$ were separated electrophoretically using 10\% SDS-PAGE and the gel was then transferred to a $0.45 \mu \mathrm{m}$ PVDF membrane. Blots were soaked in blocking buffer (5\% non-fat milk) and then incubated with primary antibodies (anti-JAK, 1:200; anti-STAT3, 1:200; anti- $\beta$-actin, $1: 1,000)$ overnight at $4{ }^{\circ} \mathrm{C}$. Following thorough washing with TBST buffer, horseradish peroxidase conjugated secondary antibodies $(1: 10,000)$ were applied and immune complexes were then visualized using the enhanced chemiluminescence detection system.

Radioimmunoassay. Levels of TNF- $\alpha$ and IL-1 $\beta$ in myocardial tissue were determined by radioimmunoassay. Myocardial tissue $(100 \mathrm{mg})$ was mixed with a 3 -fold volume of PBS and homogenized, then centrifuged at $12900 \mathrm{x}$ g for $20 \mathrm{~min}$ at $4^{\circ} \mathrm{C}$. Protein levels of TNF- $\alpha$ and IL-1 $\beta$ in the supernatant were quantified using a radioimmunoassay assay kit.

Statistical analysis. Results were presented as the mean \pm SEM of at least three separate experiments. Data were analyzed by one-way ANOVA, followed by the Student-Newman-Keuls post hoc test. $\mathrm{P}<0.05$ was considered to indicate a statistically significant difference.

\section{Results}

Cardiac function assay. Changes in myocardial diastolic function are an important characteristic of septic shock (16). A rat model of CLP was used to evaluate the effect of OMT on cardiac function in rats with septic shock. As demonstrated in Table I and Fig. 1, cardiac function parameters, including HR, MAP, LVSP, LVEDP, LVdp/dtmax and -LVdp/dtmax indices were not affected in the OMT control group compared with the control (CON) group. In the CLP group, significant changes in all indices were identified $(\mathrm{P}<0.01)$ compared with $\mathrm{CON}$. 
Table I. Effect of OMT on cardiac function in rats with septic shock (mean $\pm \mathrm{SD} ; \mathrm{n}=8$ )

\begin{tabular}{|c|c|c|c|c|c|c|}
\hline Group & $\begin{array}{l}\text { Dose } \\
(\mathrm{mg} / \mathrm{kg})\end{array}$ & HR & MAP & LVSP & LVEDP & $\pm \mathrm{LVdp} / \mathrm{dtmax}$ \\
\hline $\mathrm{CON}$ & & $398.61 \pm 11.30$ & $106.80 \pm 8.04$ & $157.90 \pm 11.27$ & $\begin{array}{c}22.64 \pm 1.78 \\
-\end{array}$ & $\begin{array}{l}5352.41 \pm 220.30 / \\
4254.40 \pm 184.02\end{array}$ \\
\hline OMT control & 26 & $410.30 \pm 10.66$ & $102.41 \pm 12.52$ & $150.92 \pm 11.76$ & $\begin{array}{c}22.21 \pm 3.01 \\
-\end{array}$ & $\begin{array}{l}5876.43 \pm 237.64 / \\
4658.64 \pm 129.54\end{array}$ \\
\hline CLP & & $452.42 \pm 12.34$ & $72.11 \pm 6.02$ & $119.74 \pm 10.63$ & $31.32 \pm 2.24$ & $\begin{array}{r}3310.70 \pm 296.08 / \\
-2879.22 \pm 375.54\end{array}$ \\
\hline CLP + OMT-L & 13 & $437.60 \pm 11.59$ & $78.80 \pm 7.32$ & $123.6 \pm 9.73$ & $30.7 \pm 1.16$ & $\begin{array}{c}3755.91 \pm 228.40 \\
-3134.20 \pm 225.34\end{array}$ \\
\hline CLP + OMT-M & 26 & $420.54 \pm 13.42$ & $84.11 \pm 9.09$ & $131.42 \pm 10.36$ & $26.80 \pm 2.93$ & $\begin{array}{c}4459.41 \pm 146.35 / \\
-3481.04 \pm 148.30\end{array}$ \\
\hline CLP + OMT-H & 52 & $402.61 \pm 15.39$ & $91.10 \pm 8.14$ & $143.31 \pm 11.58$ & $24.90 \pm 1.16$ & $\begin{array}{c}4967.21 \pm 101.53 / \\
-3605.70 \pm 415.43\end{array}$ \\
\hline CLP + DEX & 10 & $409.31 \pm 12.22$ & $90.52 \pm 8.06$ & $139.10 \pm 10.74$ & $24.11 \pm 1.25$ & $\begin{array}{c}4010.70 \pm 184.02 / \\
-3795.70 \pm 652.99\end{array}$ \\
\hline
\end{tabular}

$\mathrm{P}<0.01$ vs. CON; $\mathrm{P}<0.01$ vs. CLP; $\mathrm{P}<0.05$ vs. CLP; $\mathrm{P}<0.05$, vs. CLP+DEX. OMT, oxymatrine; CLP, cecal ligation and puncture; DEX, dexamethasone; HR, heart rate; MAP, mean arterial pressure; LVdp/dt max, left intraventricular pressure change rate; LVESP, left ventricular contraction to the end of pressure; LVEDP, left ventricular end diastolic pressure; CON, control.
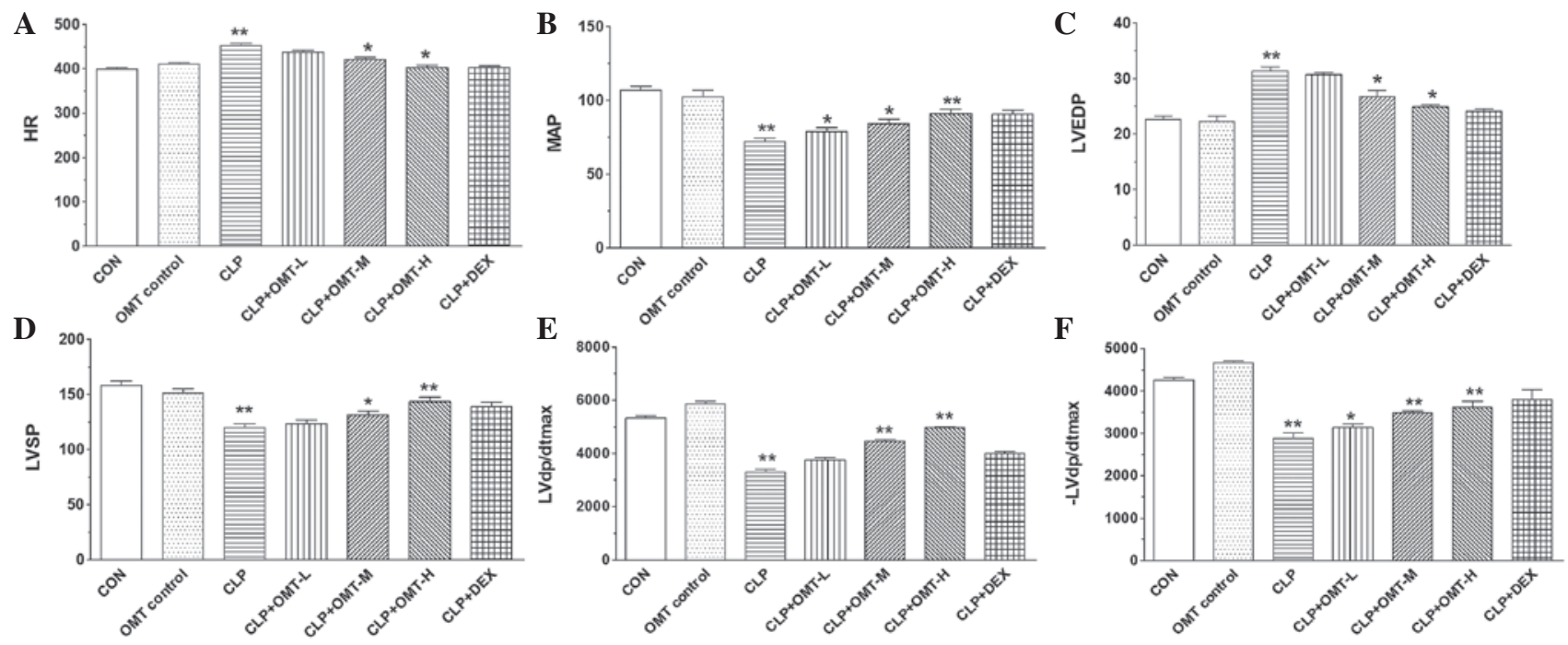

Figure 1. Effect of OMT on cardiac function in rats with septic shock (n=8). Indexes (A) HR, (B) MAP, (C) LVEDP, (D) LVSP, (E) LVdp/dtmax and (F) -LVdp/dtmax indices were measured by inserting the cardiac catheterization in all animal groups, including CON, OMT CON, CLP, CLP + L, CLP + M, CLP + H, CLP + DEX group. ${ }^{*} \mathrm{P}<0.05$ and ${ }^{* *} \mathrm{P}<0.01$, vs. CON. OMT, oxymatrine; CLP, cecal ligation and puncture; DEX, dexamethasone; HR, heart rate; MAP, mean arterial pressure; LVdp/dt max, left intraventricular pressure change rate; LVESP, left ventricular end systolic pressure; LVEDP, left ventricular end diastolic pressure; CON, control.

HR increased by $15 \%$, MAP reduced by $33 \%$, LVSP reduced by $24 \%$, LVEDP increased by $47 \%, \mathrm{LVdp} / \mathrm{dtmax}$ reduced by $38 \%$ and $-\mathrm{LVdp} / \mathrm{dtmax}$ reduced by $32 \%$. Following treatment with various doses of OMT, the CLP + high-dose OMT group was observed to reduce HR by $11 \%(\mathrm{P}<0.05)$, increase MAP by $26 \%(\mathrm{P}<0.01)$, increase LVSP by $20 \%(\mathrm{P}<0.01)$, reduce LVEDP by $20 \%(\mathrm{P}<0.01)$ and increase LVdp/dtmax by $50 \%$ $(\mathrm{P}<0.01)$ and $-\mathrm{LVdp} /$ dtmax by $25 \%(\mathrm{P}<0.01)$ compared with CLP. The CLP + middle dose OMT group was observed to have a reduced HR by $7 \%(\mathrm{P}<0.05)$, increased MAP by $17 \%$ $(\mathrm{P}<0.05)$, increased LVSP in $10 \%(\mathrm{P}<0.05)$, reduced LVEDP by $14 \%(\mathrm{P}<0.05)$ and increased LVdp/dtmax by $35 \%(\mathrm{P}<0.01)$ and $-\mathrm{LVdp} / \mathrm{dtmax}$ by $25 \%(\mathrm{P}<0.01)$. HR was reduced by $3 \%$ $(\mathrm{P}>0.05)$, MAP was increased by $9 \%(\mathrm{P}<0.05)$, LVSP was increased by $3 \%$ ( $\mathrm{P}>0.05)$, LVEDP was reduced by $2 \%(\mathrm{P}>0.05)$, LVdp/dtmax was increased by $13 \%(\mathrm{P}<0.05)$ and $-\mathrm{LVdp} /$ dtmax was increased by $9 \%(\mathrm{P}<0.05)$ in the CLP + low-dose OMT group. The positive control CLP + DEX group did not 

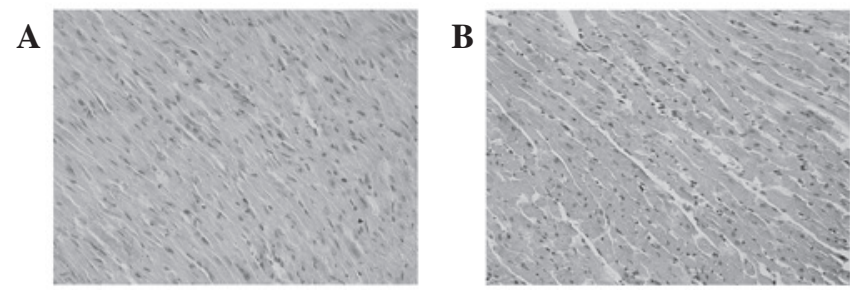

$\mathbf{E}$

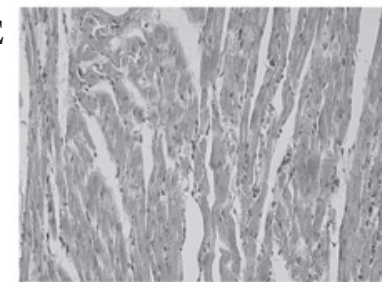

$\mathbf{F}$

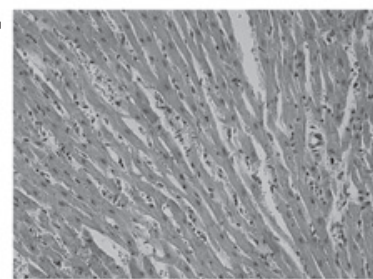

C

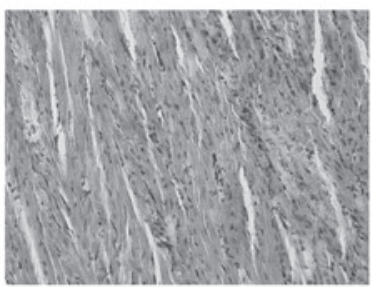

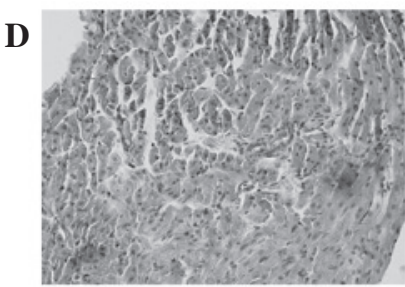

G

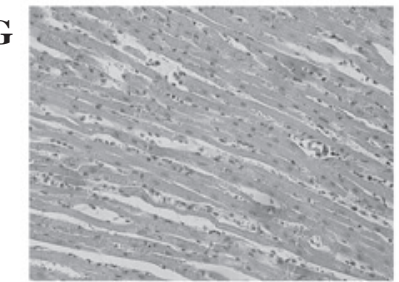

Figure 2. Myocardial histological analyses in rats with septic shock. Apical heart tissue blocks $\left(\sim 2 \mathrm{~mm}^{3}\right)$ were collected and fixed in $10 \%$ formalin, embedded in paraffin and stained with haematoxylin-eosin for histological analyses. (A) CON and sham surgery rats received intravenous injection of normal saline. (B) OMT control rats received intravenous injection of $26 \mathrm{ml} / \mathrm{kg}$ OMT. (C) CLP rats received intravenous injection of normal saline (26 ml $/ \mathrm{kg}$ ). (D) CLP + OMT-L and CLP rats received tail vein injection with $13 \mathrm{mg} / \mathrm{kg}$ OMT. (E) CLP + OMT-M and CLP rats received tail vein injection with $26 \mathrm{mg} / \mathrm{kg}$ OMT. (F) CLP + OMT-H and CLP rats received tail vein injection with $52 \mathrm{mg} / \mathrm{kg}$ OMT. (G) CLP + DEX and positive control CLP rats received tail vein injection with $10 \mathrm{mg} / \mathrm{kg}$ DEX (magnification, x200). OMT, oxymatrine; CLP, cecal ligation and puncture; DEX, dexamethasone; CON, control.

reveal marked differences in various indices compared with CON, CLP + high-dose OMT and CLP + middle-dose OMT, however a significant difference in all indices was found when compared with CLP and CLP + low-dose OMT.

Myocardial histological assay. Differences in myocardial tissue were not identified between the CON and OMT control group. The endocardial membrane was complete, with no edema and fibrous connective tissue hyperplasia. The myocardial stripes were clear, the nucleus centered and no vasodilation and inflammatory cell infiltration were observed. Epicardial membrane demonstrated integrity in the stroma without inflammatory exudate (Fig. 2A and B). As shown in Fig. 2C, CLP rat myocardial tissue revealed marked subendocardial myocardial structural disorder compared with the CON group. Infiltration of a number of inflammatory cells with a considerable number of mononuclear cells and a few lymphocytes and neutrophils and telangiectasia and bleeding were observed. The CLP group also exhibited interstitial edema, fibroblast proliferation and cell necrosis and fibrosis to various degrees. Following various doses of OMT treatment, myocardial tissue damage in the CLP + OMT-L group was reduced compared with CLP, however, low levels of disorganized myocardial structures with inflammatory cell infiltration, telangiectasia and bleeding, as well as cell necrosis and fibrosis were identified (Fig. 2D). However, myocardial tissue damage in the CLP + OMT-M and CLP + OMT-H groups was markedly reduced and a normal basic cardiac structure was observed (Fig. 2E and F). Edema, degeneration and necrosis were significantly reduced but remained accompanied by a small amount of inflammatory cell infiltration and exudative changes. Compared with the CLP group, myocardial injury was significantly reduced in the CLP + DEX group and myocardial cells were organized in rows, accompanied by a small amount of inflammatory cell infiltration. However, no significant changes in cell swelling, degeneration and necrosis were identified (Fig. 2G).

The results indicate that OMT may reduce myocardial injury and have a protective effect on cardiac structure and function in rats with septic shock. Results in the CLP + OMT groups with high and middle doses and the positive control (CLP + DEX group) were consistent.

Myocardial ultramicro-histological changes. Compared with $\mathrm{CON}$, myocardial tissue, including myofilaments, sarcomere, capillaries, mitochondrion, sarcoplasmic reticulum and nucleolus in the OMT control group were observed to exhibit no significant changes and all had normal shapes and clear structures (Fig. 3A and B). Myofilaments and sarcomere arrangement was normal and blood flow volume was normal. Mitochondrial structure was normal and clear with complete membranes, dense ridges and a clear matrix. The intercalated disc was in order and successive. The sarcoplasmic reticulum was smooth and continuous. The nucleolus was clear with visible light nucleus pycnosis and the chromatin was uniform. However, the ultramicro-histological myocardial tissue of the CLP group was observed to be significantly damaged. As is evident in Fig. 3C, mitochondria were markedly swollen, leading to membrane damage and disordered cristae. In addition, myofilaments were dissolved and sarcomeres were disordered, leading to vacuoles. The nucleus was significantly reduces in size and the chromatin margination was observed. Dissolution of intercalated disc demonstrated discontinuity and uneven distribution. However, compared with the CLP, the injuries of ultramicro-histological myocardial tissue in CLP + OMT groups were significantly reduced (Fig. 3D-F). Myocardial fiber arrangement was normal, the majority of the mitochondrial structure was complete and ridge dense arrangement had a regular pattern. Although a section of the ridge was undefined, the arrangement was still regular, mitochondrial swelling was reduced and specific incidences of damage were not recovered fully. The CLP + OMT-L group was found to exhibit the highest levels of damage. The ultramicro-histological myocardial tissue in the CLP + DEX group was almost normal and rarely injured (Fig. 3G). Myocardial fibers were aligned, the majority of mitochondrial structures were complete, ridges were dense, regions of the ridge were undefined, fibers were arranged in 
A

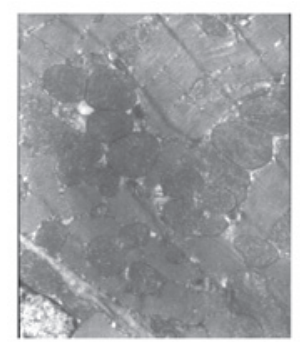

E

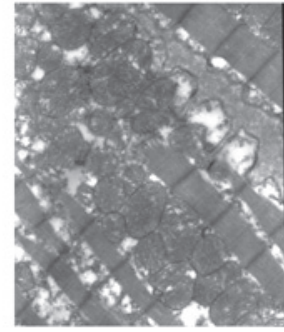

B

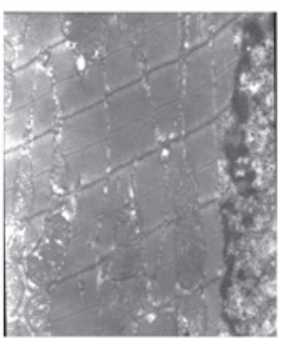

C

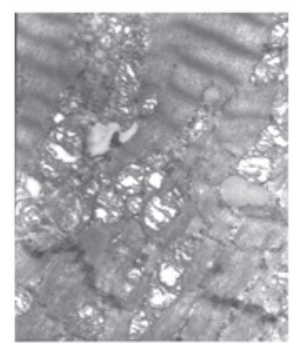

D

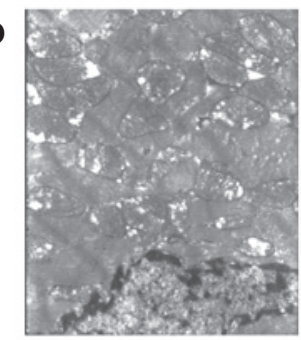

G

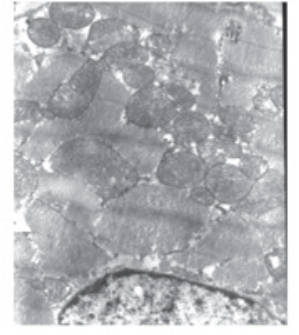

Figure 3. Myocardial ultramicro-histological analyses (x12,000). Myocardial tissues $\left(\sim 2 \mathrm{~mm}^{3}\right)$ were incubated in $2 \%$ glutaraldehyde, then sectioned as electron microscopy specimens to observe ultrastructural changes. (A) CON; (B) OMT control; (C) CLP; (D) CLP + OMT-L; (E) CLP + OMT-M; (F) CLP + OMT-H; and (G) CLP + DEX. OMT, oxymatrine; CLP, cecal ligation and puncture; DEX, dexamethasone; CON, control.

A

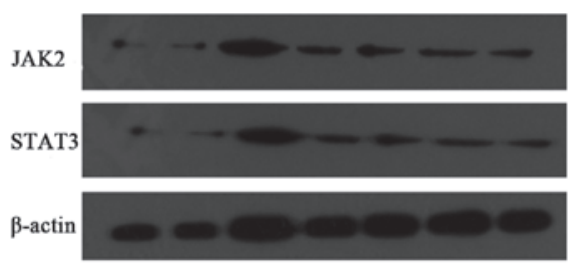

F

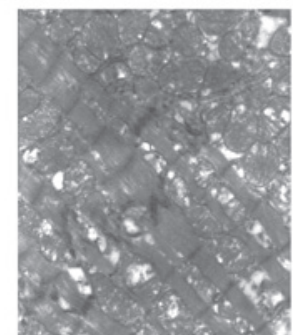

B

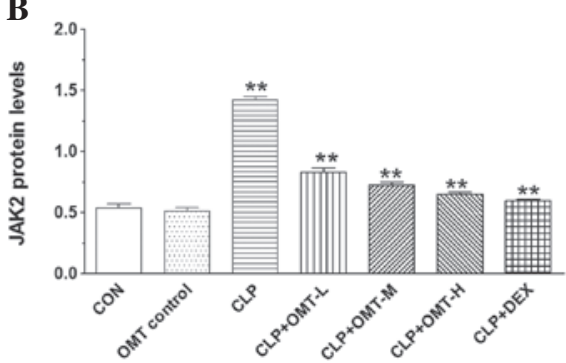

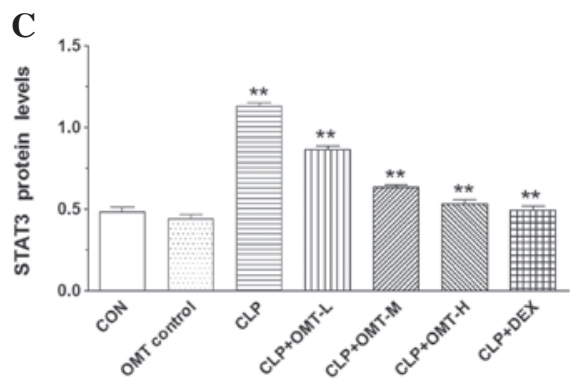

Figure 4. Effect of OMT on JAK2 and STAT3 protein expression in rat cardiac muscle with septic shock. (A) Western blot analysis of JAK2 and STAT3 protein expression, with $\beta$-actin as the internal control. Quantification of (B) JAK2 and (C) STAT3 protein expression. Lanes 1-7 show CON, OMT control, CLP, CLP + OMT-L, CLP + OMT-M, CLP + OMT-H and CLP + DEX groups, respectively. ${ }^{*} \mathrm{P}<0.05$ and ${ }^{* *} \mathrm{P}<0.01$ vs. CON. OMT, oxymatrine; CLP, cecal ligation and puncture; DEX, dexamethasone; JAK-STAT, janus kinase-signal transducer and activator of transcription; CON, control.

A

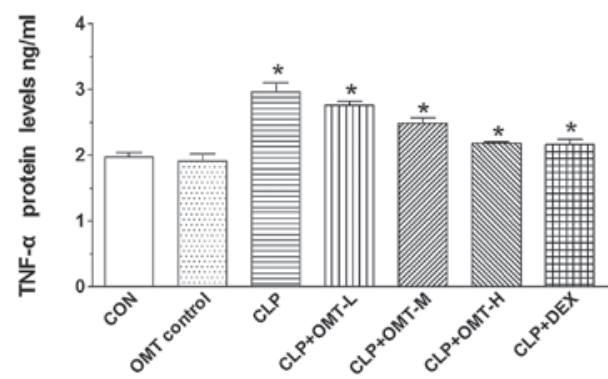

B

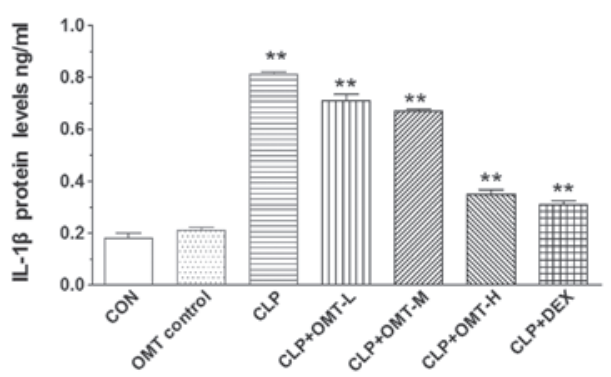

Figure 5. Effect of OMT on TNF- $\alpha$ and IL-1 $\beta$ protein expression in rat cardiac muscle with septic shock. (A) Radioimmunoassay of TNF- $\alpha$ protein levels. (B) Radioimmunoassay of IL- $1 \beta$ protein levels. ${ }^{*} \mathrm{P}<0.05$ and ${ }^{* *} \mathrm{P}<0.01$ vs. CON. OMT, oxymatrine; TNF- $\alpha$, tumor necrosis factor- $\alpha$; IL-1 $\beta$, interleukin- $1 \beta$; CON, control.

an organized manner, specific incidences of damage were not recovered fully and intercalated disc demonstrated clear continuity. The sarcoplasmic reticulum was smooth and continuous. The nucleolus was clear with light nucleus pycnosis and the chromatin was uniform.

Effect of OMT on JAK2 and STAT3 protein expression. Western blot analysis revealed that JAK2 and STAT3 protein levels in OMT control myocardial tissue was similar to that of the CON group but was observed to be significantly higher in the CLP group $(\mathrm{P}<0.05)$. However, JAK2 and STAT3 protein levels in CLP + OMT and positive control groups were markedly decreased compared with CLP $(\mathrm{P}<0.05$; Fig. 4).

Effect of OMT on TNF- $\alpha$ and IL-1 $\beta$ protein expression. Radioimmunoassay revealed that TNF- $\alpha$ and IL-1 $\beta$ protein 
A

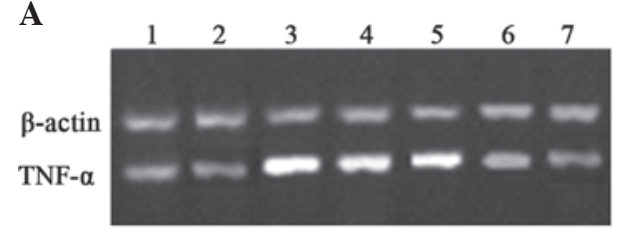

C

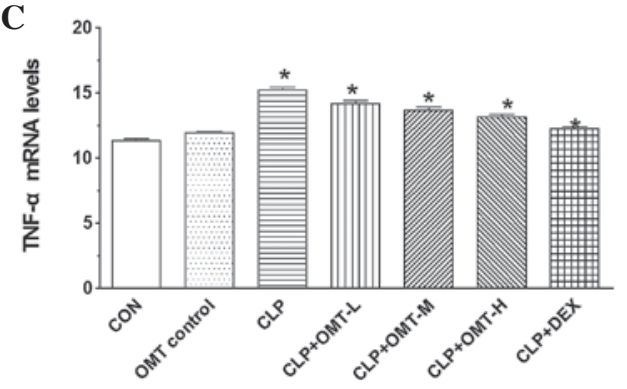

B

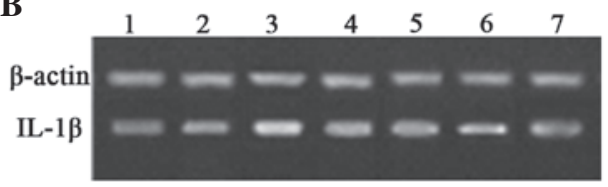

D

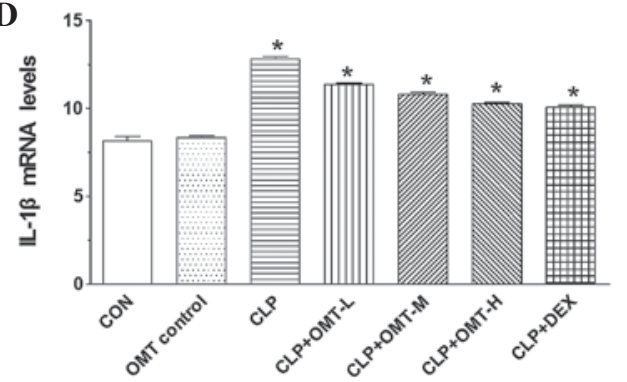

Figure 6. Effect of OMT on TNF- $\alpha$ and IL-1 $\beta$ mRNA expression in rat cardiac muscle with septic shock. RT-PCR of (A) TNF- $\alpha$ and (B) IL-1 $\beta$ mRNA expression. M, marker; lanes 1-7, CON, OMT control, CLP, CLP + OMT-L, CLP + OMT-M, CLP + OMT-H and CLP + DEX groups, respectively. Length of PCR product for TNF- $\alpha$, IL-1 $\beta$ and $\beta$-actin were 355, 399 and 662 bp, respectively. Quantification of (C) TNF- $\alpha$ and (D) IL-1 13 mRNA levels. "P<0.05 vs. CON. OMT, oxymatrine; CLP, cecal ligation and puncture; DEX, dexamethasone; TNF- $\alpha$, tumor necrosis factor- $\alpha$; IL- $1 \beta$, interleukin- $1 \beta$; CON, control.

levels in the CLP group was significantly increased compared with the CON and OMT control $(\mathrm{P}<0.05)$. However, compared with the CLP group, TNF- $\alpha$ and IL- $1 \beta$ protein levels in CLP + OMT and the positive control were found to be significantly decreased ( $\mathrm{P}<0.05 ;$ Fig. 5$)$.

Effect of OMT on TNF- $\alpha$ and IL-1 $\beta$ mRNA expression. TNF- $\alpha$ and IL-1 $\beta$ mRNA levels in OMT control and CON myocardial tissue were normal, however, levels were identified to be significantly increased in the CLP group $(\mathrm{P}<0.05)$. Compared with the CLP group, TNF- $\alpha$ and IL- $1 \beta$ mRNA levels in $\mathrm{CLP}+\mathrm{OMT}$ and the positive control were markedly decreased $(\mathrm{P}<0.05$; Fig. 6).

\section{Discussion}

Septic shock is a serious complication in acutely ill patients suffering from severe trauma, burns, shock, infection and major surgery. The widespread activation of cells in septic shock results in the release of a number of inflammatory mediators, including cytokines, chemokines, prostaglandins, lipid mediators and reactive oxygen species. Excess production of inflammatory mediators in sepsis is associated with numerous signaling pathways, including MAPK and NF- $\mathrm{KB}$ and in particular, JAK/STAT (17-22). JAK2 and STAT1/3 promote the release of cytokines, including TNF- $\alpha$ and IL- $1 \beta$ and are markedly associated with the uncontrolled inflammatory response, septic shock and acute organ injury (23). Wang et al reported that IL-2 and IFN- $\gamma$ are directly associated with the JAK/STAT pathway activation and inhibition of this pathway reduces dysfunction of vital organs in septic shock in rats (16). An extremely limited number of studies have analyzed the role of the JAK/STAT signaling pathway in septic shock.

OMT is an alkaloid extracted from the Sophora flavescens Ait and its bioactivities have been studied extensively (24-26). OMT has been found to attenuate hepatic fibrosis via targeting hepatic stellate cells and reducing bleomycin-induced pulmonary fibrosis in mice via inhibition of the inducible nitric oxide synthase expression and the TGF- $\beta$ /Smad signaling pathway (24-26). In addition, OMT was found to exhibit a neuroprotective effect in cerebral ischemia/reperfusion. However, the pharmacological effects of OMT on cardiac tissues and the mechanisms remain unknown.

Our previous study revealed that OMT significantly inhibited JAK2 and STAT3 activity in septic rat lung tissue, decreased TNF- $\alpha$ and IL-6 levels, W/D ratio and pulmonary coefficient and reduced lung tissue congestion, edema, neutrophil infiltration, hyaline membrane formation and other lesions (7). In the present study, septic shock rat models were developed to examine the effect of OMT on cardiac tissue injuries and the JAK/STAT signaling pathway by detecting JAK2 and STAT3 expression and the release of TNF- $\alpha$ and IL-6. Histological analysis revealed that OMT intervention markedly reduced septic shock in rats caused by increases in HR and LVSP. Decreased \pm LVdp/dtmax increased myocardial compliance, reduced myocardial stiffness, improved heart function, enhanced myocardial contraction force, corrected ventricular end-diastolic pressure increases and reduced preload. These cardiac protective effects are associated with activation of the JAK/STAT signaling pathway. Western blot analysis and RT-PCR revealed that OMT inhibits the activation of JAK2 and STAT3 leading to a reduced expression of proinflammatory cytokines, TNF- $\alpha$ and IL-6. These results are consistent with our previous study (27).

This is the first study to demonstrate that cardiac injuries are associated with the JAK/STAT signaling pathway and OMT has the ability to suppress the activation of this signaling pathway. Activation of JAK2 and STAT3 leads to the upregulation of TNF- $\alpha$ and IL-6. Release of TNF- $\alpha$ and IL- 6 has marked effects on cardiac tissue. Current observations indicate that OMT protects myocardial injury and is associated, at least in part, with significant inhibition of JAK2/STAT3 signaling in rats with septic shock and may be a potential therapeutic agent for the treatment of septic shock. 
In summary, the present results provide valuable insight into the mechanisms underlying the cardiac protective effects of OMT and the possible application of OMT for the treatment of inflammatory diseases accompanied by septic shock.

\section{Acknowledgements}

The present study was supported by grants from the Natural Science Foundation of Ningxia (NZ1194), National Natural Science Foundation of China (30960108, 31060140 and 31260243), 2012 Yinchuan Key Scientific and Technological Project for Minghao Zhang, Key Project of Department of Public Health of Ningxia (2012004) and the project-sponsored by SRF for ROCS, SEM for Yin Wang.

\section{References}

1. Van Amersfoort ES, Van Berkel TJC and Kuiper J: Receptors, mediators and mechanisms involved in bacterial sepsis and septic shock. Clin Microbiol Rev 16: 379-414, 2003.

2. Kumar A, Haery C and Parrillo JE: Myocardial dysfunction in septic shock. Crit Care Clin 16: 251-287, 2000

3. Cao W, Wang Y, Lv X, Yu X, Li X, Li H, Wang Y, Lu D, Qi R and Wang $\mathrm{H}$ : Rhynchophylline prevents cardiac dysfunction and improves survival in lipopolysaccharide-challenged mice via suppressing macrophage $\mathrm{I}-\kappa \mathrm{B} \alpha$ phosphorylation. Int Immunopharmacol 14: 243-251, 2012.

4. Celes MR, Prado CM and Rossi MA: Sepsis: going to the heart of the matter. Pathobio 80: 70-86, 2012.

5. Mao YJ, Li HH, Li JF and Shen JS: Signal transduction by protein tyrosine kinases and antitumor agents. Yao Xue Xue Bao 43: 323-334, 2008 (In Chinese).

6. Liu X, Ye L, Bai Y, Mojidi H, Simister NE and Zhu X: Activation of the JAK/STAT-1 signaling pathway by IFN-gamma can down-regulate functional expression of the MHC class I-related neonatal Fc receptor for IgG. J Immunol 181: 449-463, 2008.

7. Zhang MH, Li GZ and Cao J: Effect of oxymatrine on JAK/ STAT iteral in rat lung tissue with sepsis. Zhongguo Zhong Yao Za Zhi 35: 103-107, 2010 (In Chinese).

8. Matsukawa A: STAT proteins in innate immunity during sepsis lessons from gene knockout mice. Acta Med Okayama 5: 239-245, 2007.

9. Yuan X, Wang Y, Du D, Hu Z, Xu M, Xu M and Liu Z: The effects of the combination of sodium ferulate and oxymatrine on lipopolysaccharide-induced acute lung injury in mice. Inflammation 35: 1161-1168, 2012.

10. Cui HL, Wang YF, Li XL and Kang QX: Clinical observation of matrine injection in the treatment of 51 cases of various types of cancers. Shanxi Med J 22: 232-233, 1993.

11. Shi GF and Li Q: Effects of oxymatrine on experimental hepatic fibrosis and its mechanism in vivo. World J Gastroenterol 11: 268-271, 2005

12. Zheng P, Niu FL, Liu WZ, Shi Y and Lu LG: Anti-inflammatory mechanism of oxymatrine in dextran sulfate sodium-induced colitis of rats. World J Gastroenterol 11: 4912-4915, 2005.
13. Xu GL, Yao L, Rao SY, Gong ZN, Zhang SQ and Yu SQ: Attenuation of acute lung injury in mice by oxymatrine is associated with inhibition of phosphorylated p38 mitogen-activated protein kinase. J Ethnopharmacol 98: 177-183, 2005.

14. Han Y, Zhou Y and Liu Q: Antiendotoxic effects of Sophora alopecuroides L. Zhong Yao Cai 29: 1066-1068, 2006 (In Chinese).

15. Jing HM: A model of sepsis in rat after a cecal ligation and puncture. Chin J Pathophysiol 6: 126-127, 1990 (In Chinese).

16. Wang SB, Yao YM and Chen JS: Relationship between activation of Janus kinase/signal transducer and activator of transcription pathway and multiple organ dysfunction in rats with sepsis. Infection Inflammation Repair 29: 42-44, 2004 (In Chinese).

17. Risco A, del Fresno C, Mambol A, Alsina-Beauchamp D, MacKenzie KF, Yang HT, Barber DF, Morcelle C, Arthur JS, Ley SC, Ardavin C and Cuenda A: p38 $\gamma$ and $\mathrm{p} 38 \delta$ kinases regulate the Toll-like receptor 4 (TLR4)-induced cytokine production by controlling ERK1/2 protein kinase pathway activation. Proc Natl Acad Sci USA 109: 11200-11205, 2012.

18. Oishi H, Takano K, Tomita K, Takebe M, Yokoo H, Yamazaki M and Hattori Y: Olprinone and colforsin daropate alleviate septic lung inflammation and apoptosis through CREB-independent activation of the Akt pathway. Am J Physiol Lung Cell Mol Physiol 303: 130-140, 2012.

19. Lee C, Lim HK, Sakong J, Lee YS, Kim JR and Baek SH: Janus kinase-signal transducer and activator of transcription mediates phosphatidic acid-induced interleukin (IL)-1beta and IL-6 production. Mol Pharmacol 69: 1041-1047, 2006.

20. Qian F, Deng J, Gantner BN, Flavell RA, Dong C, Christman JW and Ye RD: Map kinase phosphatase 5 protects against sepsis-induced acute lung injury. Am J Physiol Lung Cell Mol Physiol 302: 866-874, 2012.

21. Krüttgen A and Rose-John S: Interleukin-6 in sepsis and capillary leakage syndrome. J Interferon Cytokine Res 32: 60-65, 2012.

22. Hui L, Yao Y, Wang S, Yu Y, Dong N, Li H and Sheng Z: Inhibition of Janus kinase 2 and signal transduction and activator of transcription 3 protect against cecal ligation and puncture-induced multiple organ damage and mortality. J Trauma 66: 859-865, 2009.

23. Gu XB, Yang XJ, Hua Z, Lu ZH, Zhang B, Zhu YF, Wu HY, Jiang YM, Chen HK and Pei H: Effect of oxymatrine on specific cytotoxic T lymphocyte surface programmed death receptor-1 expression in patients with chronic hepatitis B. Chin Med J (Engl) 125: 1434-1438, 2012.

24. Fan DL, Zhao WJ, Wang YX, Han SY and Guo S: Oxymatrine inhibits collagen synthesis in keloid fibroblasts via inhibition of transforming growth factor- $\beta 1 /$ Smad signaling pathway. Int J Dermatol 51: 463-472, 2012.

25. Liu L, LuW, Ma Z and Li Z: Oxymatrine attenuates bleomycin-induced pulmonary fibrosis in mice via the inhibition of inducible nitric oxide synthase expression and the TGF- $\beta / S m a d$ signaling pathway. Int J Mol Med 29: 815-822, 2012.

26. Chai NL, Fu Q, Shi H, Cai CH, Wan J, Xu SP and Wu BY: Oxymatrine liposome attenuates hepatic fibrosis via targeting hepatic stellate cells. World J Gastroenterol 18: 4199-4206, 2012.

27. Zhang MH, Wang XY and Zhang Y: The preventive and therapeutic effects of oxymatrine on cardiac muscle injury in rats with septic shock. J Ningxia Med Univ 32: 876-879, 2012 (In Chinese). 